\title{
Double Level Extension-Distraction Thoracic Spine Injuries with Concomitant Double Level Sternal Fractures: A Case Report
}

\author{
CK Lee, MBBS, CYw Chan, MS Orth (UM), LB Saw, MS Orth (UM), MK Kwan, MS Orth (UM) \\ Department of Orthopaedic Surgery, University Malaya Medical Centre, Kuala Lumpur, Malaysia
}

\begin{abstract}
Extension-distraction injury of the spine is rare. A majority of these cases occur in the cervical region and at an isolated level. Rare instances of this injury have been described in an ankylosed or fused spine. This case report describes a rare two level extension-distraction thoracic spine injury in an otherwise healthy adult resulting from a motor vehicle accident.
\end{abstract}

Key Words:

Extension-distraction Injury, Thoracic Spine Injury, Damage Control Orthopaedic, Minimally Invasive Spine Surgery, MANTIS® System

\section{INTRODUCTION}

Extension-distraction injuries caused by hyperextension of the spine typically occur in the cervical region, as seen in "whiplash" injuries. This type of injury is rare in the thoracolumbar spine, comprising less than 3\% of fractures in this region ". Denis and Burkus described it as "lumberjack paraplegia" as most patients with this injury were struck in the back by a falling tree ${ }^{2}$. Rare instances of traumatic thoracolumbar extension-distraction injuries have been described in adults with rigid spines resulting from diffuse idiopathic skeletal hyperostosis (DISH) and ankylosing spondylitis as these conditions predispose the adjacent level to fracture due to acute transition in the stiffness of the spine $^{3}$. In the majority of cases, extension distraction injuries occur at an isolated (single) level. This case report describes a rare two level extension-distraction thoracic spine injury in an otherwise healthy adult resulting from a motor vehicle accident.

\section{CASE REPORT}

A 68-year-old male driver was admitted following a motor vehicle accident. His car was hit from the back by another car at a very high speed. The impact was so high that the rear seat of his car directly hit the back of this gentleman. He was wearing a seatbelt at the time of the accident. He presented with severe chest and back pain and difficulty in breathing. There were no other injuries noted. Physical examination revealed an obese $(>100 \mathrm{~kg})$ normotensive male with tachycardia and tachypnoea. A flail chest was noted. Air entry was reduced bilaterally with generalized crepitus. No head, abdominal or neurological injury was noted. Examination of the back demonstrated tenderness over the cervicothoracic junction and midthoracic region with visible ecchymoses over the injured region.

The initial radiograph showed increased disc space between the 1st and 2nd thoracic vertebrae and 7th and 8th thoracic vertebrae. Computed Tomographic (CT) scan revealed fracture through the lower endplate of the first thoracic vertebra with increased disc space, facet joint space and interspinous space (Figure 1). There was another fracture through the upper endplate of the 8th thoracic vertebra and spinous processes of the 6th to 10th thoracic vertebrae with increased T7/T8 disc space. There were also 2 corresponding fractures of the upper and lower sternum (Figure 1). Other associated injuries were multiple rib fractures, severe lung contusions and haemothorax.

Endotracheal intubation was performed due to the severe chest injury and bilateral chest tubes were inserted. He was admitted to ICU. A minimally invasive percutaneous posterior instrumentation using the Minimal Access NonTraumatic Insertion System (MANTIS ${ }^{\circledR}$ system) was performed one day after the trauma to stabilize the fractures and to facilitate nursing care and chest physiotherapy for his severe chest injury in an ideal position (propped up at a $45^{\circ}$ angle). The MANTIS procedure was performed with the patient positioned prone on a radiolucent table. Four size $5.5 \mathrm{~mm}$ percutaneous screws were inserted simultaneously under image intensifier guidance (Figure 2). The operative time was 40 minutes and entailed minimal blood loss. The upper fracture could not be stabilized percutaneously due to difficulty in obtaining satisfactory image intensifier images. The patient was also difficult to ventilate in prone position and therefore the procedure has to be shortened. A cervical orthosis was applied as a temporary measure while waiting 


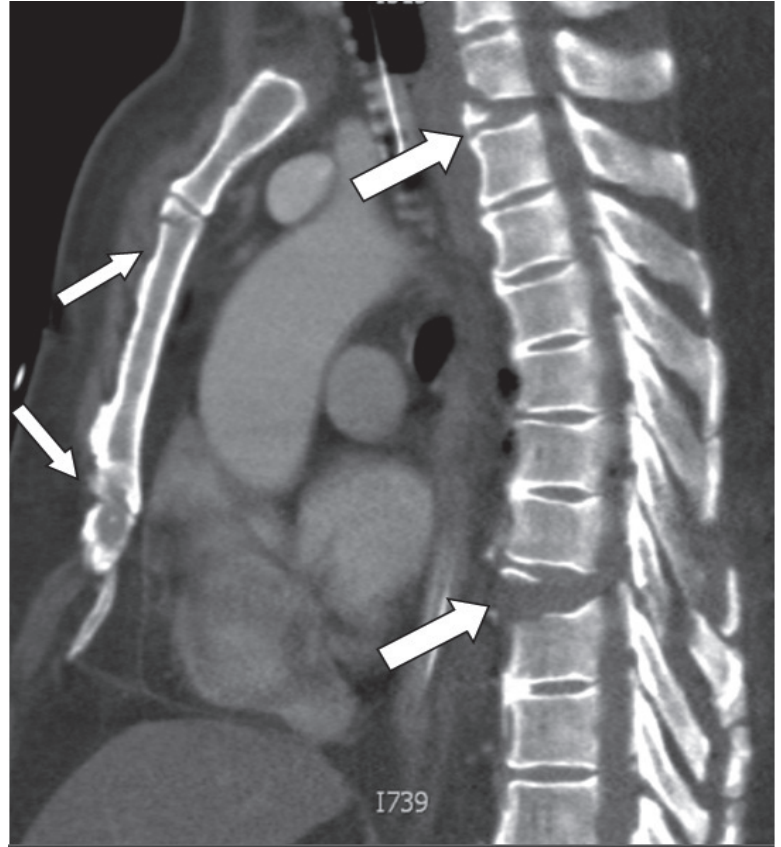

Fig. 1: The upper thick arrow shows the fracture through lower endplate of first thoracic vertebra with increased disc space. The lower thick arrow shows another fracture through the upper endplate of 8th thoracic vertebra and spinous process of 6 th to 10th thoracic vertebrae with increased T7/T8 disc space. The thin arrows show the two corresponding fractures at the upper and lower sternum.

for the chest injury to resolve.

Unfortunately, in this case, despite the early stabilization of the thoracic spine, the patient succumbed to the extensive chest injuries, which progressed to adult respiratory distress syndrome and multi-organ failure.

\section{DISCUSSION}

Traumatic extension-distraction injuries of the spine are very rare. The majority of cases occur in the cervical spine leading to central cord syndrome due to the projection of the osteophytes and ligamentum flavum onto the spinal cord ${ }^{1}$. As compared to the cervical spine, the thoracic spine is less mobile because of its connection to the chest wall. This restricted mobility reduces the probability of hyperextension that occurs in extension-distraction injury. Therefore the aetiology of this type of thoracic spine fracture is usually attributed to two factors.

The first factor, widely described in the English literature, is the occurrence of extension distraction injury of the thoracic spine in the setting of an ankylosed or fused spine. In such situations, a long segment of an extremely rigid spine focuses the stress onto the remaining motion segment therefore predisposing that segment to fractures. This explains the thoracic extension-distraction injury, which is described in diffuse idiopathic skeletal hyperostosis (DISH) and ankylosing spondylitis ${ }^{3}$. On the other hand, in the

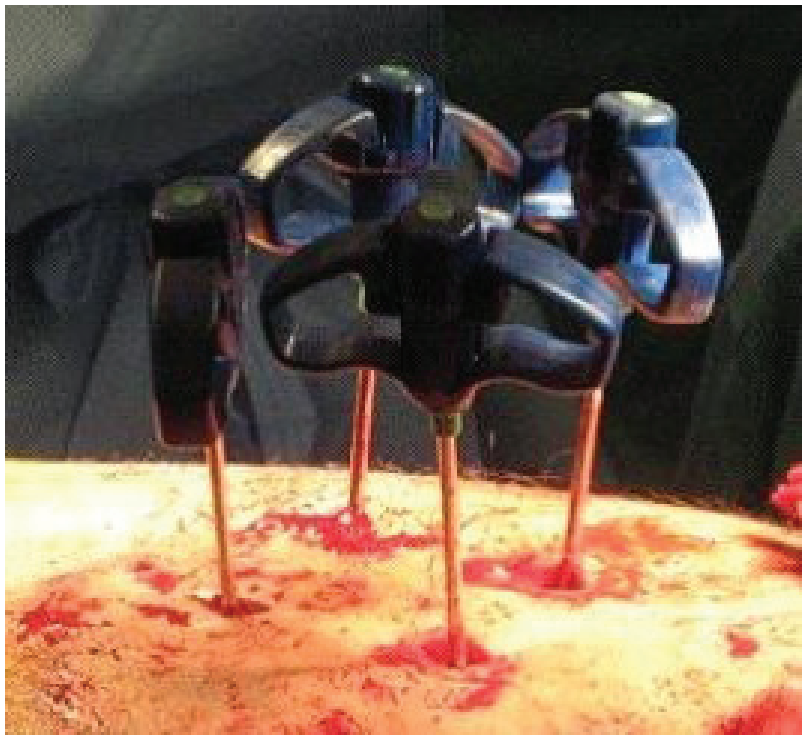

Fig. 2: Simultaneous insertion of the pedicle probes for percutaneous stabilization of the spine using the MANTIS system.

absence of this predisposing factor, the amount of force needed to cause this injury in the thoracic spine is quite significant as the thoracic spine is guarded by the rib cage as well as the sternum. This is the reason that this type of injury is sometimes described as "lumberjack paraplegia" as many patients presenting with this injury have been struck in the back by a falling tree ${ }^{2}$.

The mechanism of injury in extension distraction injuries of the thoracic spine is actually the reverse of that seen in Chance fractures, in which excessive forward flexion with the seat belt or dashboard acting as the fulcrum leads to three column disruption of the spine leading to a highly unstable fracture. In extension distraction injuries, as in the present case, we hypothesized that the mechanism of injury was due to a direct, high impact force coming posteriorly and causing acute hyperextension of the spine with the rear seat acting as a fulcrum. The rear seat had a broad surface and therefore its edges acted as two separate fulcrums during the high impact contact with the patient. As a result, the patient sustained two non-contiguous extension-distraction injuries (at different levels) of thoracic spine. The amount of energy transferred during the accident is tremendous. This explains why in such cases, viscera injuries (such as the lung or the intraabdominal organs) are common and may be life threatening. In the spine, extension-distraction injuries cause anterior disruption through the vertebral body or intervertebral disc with avulsion of the vertebral body and osteophytes and are accompanied by posterior column bony or ligamentous 
disruption ${ }^{4}$. Computed tomography findings in this patient (Figure 1) confirmed the description of this injury.

The visceral injuries together with the unstable spine fractures can lead to a management dilemma. Traditionally, these fractures are treated with open reduction and instrumented stabilisation with or without decompression. This involves long surgical duration and possibly extensive blood loss. In such cases, this is less than ideal due to the additional insults that the surgery inflicts upon the patient.

As an analogy, in appendicular skeletal trauma, the concept of damage-control orthopaedics (DCO) has evolved over the years and has become widely accepted as the key principle in the initial management for polytraumatised patients ${ }^{5}$. Fractures are temporarily stabilised using external fixators, which limit the blood loss. At the same time, this allows stabilisation of the fractures, which in turn facilitates nursing care. However, in spinal trauma, this concept could not be implemented previously due to the nature of open spine surgery. With recent advances in Minimally Invasive Spine Surgery (MISS), the percutaneous methodology allows stabilisation of the spinal column when open surgery cannot be performed ${ }^{6}$. MISS may minimize any additional trauma caused by the surgery. As this patient also sustained severe chest injury and was intubated, nursing and chest physiotherapy became crucial for his recovery. With stabilization using MISS, he could be propped up and thus undergo effective physiotherapy which would enhance his chance of full recovery.

This case illustrates a rare two level extension distraction injury with a concomitant two level sternal fracture that occurred in a previously normal spine. Such fractures are typically associated with severe visceral injuries. Percutaneous stabilisation of the spine offers a chance to stabilise such fractures without additional insult to the patient in keeping with 'damage control surgery'. 


\section{REFERENCES}

1. Burke DC. Hyperextension injuries of the spine. J Bone Joint Surg Br 1971; 53: 3-12.

2. Denis F, Burkus JK. Shear fracture-dislocations of the thoracic and lumbar spine associated with forceful hyperextension (lumberjack paraplegia). Spine 1992; 17: 156-61.

3. Burkus JK, Denis F. Hyperextension injuries of the thoracic spine in diffuse idiopathic skeletal hyperostosis. Report of four cases. J Bone Joint Surg Am 1994; 76: 237-43.

4. Dorr LD, Harvey JP Jr, Nickel VL. Clinical review of the early stability of spine injuries. Spine 1982; 7: 545-50.

5. Robert CS, Pape HC, Jones AL, Malkani AL, Rodriguez JL, Giannoudis PV. Damage control orthopaedics: Evolving concepts in the treatment of patients who have sustained orthopaedic trauma. J Bone Joint Surg Am 2005; 87: 434-49.

6. Poelstra K, Gelb D, Kane B, Ludwig S. The feasibility of damage control spine surgery minimally invasive spinal stabilization (MISS) in the acute setting for complex thoracolumbar fractures. Spine J 2008; 8: 89S. 